\section{Factors affecting outcomes of corneal collagen crosslinking treatment}

\begin{abstract}
Purpose To assess the effects of preoperative patient characteristics on clinical outcomes of corneal crosslinking (CXL) treatment in patients with progressive keratoconus.

Methods This retrospective study comprised 96 eyes of 96 patients who had unilateral CXL treatment for progressive keratoconus.

All patients underwent a complete ophthalmological examination and corneal topography at baseline and 1 year. Subgroup analyses were performed according to the age
\end{abstract} ( $<30$ and $\geq 30$ years), gender, preoperative corrected distance visual acuity (CDVA, $<0.3$ and $\geq 0.3 \log$ MAR (log of the minimum angle of resolution)), preoperative maximum keratometry $(K,<54$ and $\geq 54 \mathrm{D})$, baseline topographic cone location (central, paracentral, and peripheral), and preoperative thinnest pachymetry $(<450$ and $\geq 450 \mu \mathrm{m})$ to determine the associations between preoperative patient characteristics and outcomes (changes in visual acuity and maximum keratometry) of CXL treatment. Results In the entire study population, mean CDVA and maximum $K$ significantly improved after CXL treatment $(P<0.001)$. Patients with a preoperative CDVA of 20/40 Snellen equivalent or worse $(\geq 0.3 \log$ MAR) experienced more visual improvement after CXL treatment $(P<0.001)$. However, an age $\geq 30$ years and a baseline thinnest pachymetry less than $450 \mu \mathrm{m}$ were found significantly associated with more flattening in maximum keratometry $(P=0.024, P=0.005$ respectively). Gender, preoperative maximum $K$, and baseline topographic cone location did not show significant effect on postoperative visual acuity and maximum keratometry $(P>0.05)$.

Conclusions In patients with progressive keratoconus, age, baseline visual acuity, and baseline thinnest pachymetry seem to affect the success of the CXL treatment. Eye (2014) 28, 41-46; doi:10.1038/eye.2013.224; published online 18 October 2013

Keywords: corneal collagen cross-linking; keratoconus; preoperative predictors; success

\section{Introduction}

Keratoconus is a non-inflammatory ectatic corneal disorder. ${ }^{1}$ Major clinical findings are progressive stromal thinning and visual deterioration secondary to irregular astigmatism, myopia, and central corneal scarring. 1,2 Conservative treatment modalities such as spectacles and gas permeable rigid contact lenses become insufficient for visual rehabilitation in advanced stages of keratoconus and $10-20 \%$ of the patients need corneal transplantation. ${ }^{2}$

Wollensak et al ${ }^{3,4}$ introduced corneal collagen crosslinking (CXL) using riboflavin and ultraviolet A (UVA) as a new hope for treatment of progressive keratoconus. Studies concluded that CXL is an effective procedure to arrest the progression of keratoconus by increasing biomechanical stability of the cornea. ${ }^{4-6}$ Moreover, significant improvements in visual acuity and keratometry $(K)$ readings after CXL treatment could be considered as a regression in disease severity. ${ }^{7-10}$

Although the efficacy of CXL treatment has been shown in many studies, there is little evidence about the influence of preoperative patient characteristics on clinical outcomes of CXL treatment. ${ }^{8-10}$ In this study, we aimed to assess the effects of initial demographic and topographic properties on outcomes of CXL treatment in patients with progressive keratoconus at 1-year follow-up.
${ }^{1}$ Department of Ophthalmology, Servergazi State Hospital, Denizli, Turkey

${ }^{2}$ Department of Ophthalmology, Faculty of Medicine, Pamukkale University, Denizli, Turkey

${ }^{3}$ Private Yaylalı Eye Hospital, Denizli, Turkey

${ }^{4}$ Private Ege Akademi Eye Hospital, Denizli, Turkey

Correspondence:

$\checkmark$ Yaylalı, Department of Ophthalmology, Faculty of Medicine, Pamukkale University, Yaylalı Göz Hastanesi, Sırakapılar Mahallesi 495 Sokak No:21, Denizli 20010, Turkey.

Tel: +90 25826410 30;

Fax: +90 2582658242 .

E-mail: volkanyaylali@

yahoo.com

Received: 7 May 2013 Accepted in revised form: 31 July 2013

Published online: 18 October 2013 


\section{Materials and methods}

\section{Study population}

This retrospective study comprised 96 eyes of 96 patients who underwent unilateral CXL treatment for progressive keratoconus. The study was performed in adherence to the tenets of the Declaration of Helsinki and local ethics committee approved the methodology. Inclusion criteria were biomicroscopic examination and corneal topography consistent with keratoconus, inferiorsuperior ratio on topographic map $>1.5$, at least 18 years of age, thinnest corneal pachymetry of $\geq 400 \mu \mathrm{m}$. Progression was defined as $\geq 1 \mathrm{D}$ steepening of maximum keratometry or an increase of $\geq 1.00 \mathrm{D}$ in manifest cylinder or an increase of $\geq 0.50 \mathrm{D}$ in manifest refraction spherical equivalent in 2 years. Eyes with prior corneal surgery or scarring were excluded from the study.

\section{Examinations}

All patients underwent detailed ophthalmological examinations that included corrected (including contact lens) distance visual acuity (CDVA) measurement with Snellen charts, slit-lamp biomicroscopic examination, applanation tonometry, and dilated funduscopic examination. Visual acuity in Snellen format was converted to the logMAR (log of the minimum angle of resolution) equivalent for statistical analysis. ${ }^{11}$ The mean CDVA was noted at baseline and 1 year after CXL treatment.

New generation anterior segment tomography devices are capable of determining the topography and pachymetry of the entire cornea. ${ }^{12-14}$ In this study, corneal topography (Allegro Oculyzer, WaveLight AG, Erlangen, Germany) was conducted with undilated pupils under scotopic conditions by a single experienced technician. Scans were taken in automatic mode with proper quality. Maximum keratometry and corneal pachymetry at the thinnest location were recorded at baseline and 1 year after CXL treatment. ${ }^{13,14}$ Cone location was determined according to the topographic coordinates of maximum keratometry as follows: central cone (maximum $K$ within the central $3 \mathrm{~mm}$ optical zone), paracentral cone ( $3-5 \mathrm{~mm}$ optical zone), and peripheral cone (outside the central $5 \mathrm{~mm}$ optical zone).

Subgroups and cutoff values were created in accordance with the current literature as follows: age ( $<30$ and $\geq 30$ years), gender, preoperative CDVA $(<0.3$ and $\geq 0.3 \log$ MAR), preoperative maximum $K(<54$ and $\geq 54 \mathrm{D}$ ), baseline topographic cone location (central, paracentral, and peripheral cone), and preoperative thinnest pachymetry $(<450$ and $\geq 450 \mu \mathrm{m}$ ) to analyze the relationship between preoperative patient characteristics and improvements in CDVA and maximum $K$ after CXL treatment.

\section{Surgical procedure}

CXL was performed under sterile conditions using topical anesthesia as described by Wollensak. ${ }^{4}$ Central $9.0 \mathrm{~mm}$ corneal epithelium was removed by mechanical debridement. Isotonic riboflavin solution $(0.1 \%$ in $20 \%$ dextran T500 solution) was instilled every $2 \mathrm{~min}$ for $30 \mathrm{~min}$ until riboflavin absorption was observed in the corneal stroma and anterior chamber under biomicroscopic examination. Then, the cornea was exposed to UVA irradiation at the wavelength of $365 \mathrm{~nm}$ and surface irradiance of $3 \mathrm{~mW} / \mathrm{cm}^{2}$. During the UVA exposure, isotonic riboflavin solution instillation was continued every $2 \mathrm{~min}$ for $30 \mathrm{~min}$. Postoperatively, a soft contact lens was placed until corneal epithelial healing completed. Topical antibiotic and corticosteroid drops were administered and continued four times daily for 1 week and 2 weeks, respectively.

\section{Statistical analysis}

Statistical analysis was performed with the Statistical Package for Social Sciences software version 16.0 (SPSS Inc, Chicago, IL, USA). Results were expressed as mean \pm SD. A paired $t$-test was used to analyze the changes in mean CDVA and maximum $K$ between baseline and 1 year. Comparison of the quantitative data (changes in mean CDVA and maximum $K$ ) between two groups was performed by independent samples $t$-test. However, non-parametric tests (Wilcoxon signed ranks test and Mann-Whitney $U$-test) were used to analyze the effects of preoperative topographic cone location on mean CDVA and maximum $K$. A $P$-value less than 0.05 was considered statistically significant at $95 \%$ confidence interval.

\section{Results}

Ninety-six eyes of ninety-six patients who had unilateral CXL treatment for progressive keratoconus were included in this retrospective study. The study group consisted of $39(40.6 \%)$ male and $57(59.4 \%)$ female with a mean age of $29.42 \pm 9.34$ years (Table 1 ). All patients had 1 -year follow-up. In the entire cohort, mean CDVA significantly improved from $0.39 \pm 0.29 \log$ MAR $(20 / 49$ Snellen) to $0.28 \pm 0.22 \log$ MAR (20/38 Snellen; Table 2, $P<0.001)$ and the maximum $K$ significantly decreased from $54.54 \pm 5.50 \mathrm{D}$ to $53.52 \pm 5.18 \mathrm{D}$ between baseline and 1 year after CXL treatment (Table $2, P<0.001$ ). 
The effects of preoperative patient characteristics on changes in CDVA and maximum $\mathrm{K}$ after CXL treatment were assessed.

\section{Subgroup analyses according to the preoperative patient data}

Age Patients with $<30$ years of age $(n=54)$ : The mean CDVA significantly improved from baseline $0.34 \pm 0.23$ logMAR (20/43 Snellen) to $0.21 \pm 0.15 \log$ MAR (20/32 Snellen) at 1 -year after CXL treatment $(P<0.001)$, whereas improvement in maximum $K$ (from $53.62 \pm 4.90 \mathrm{D}$ to $53.06 \pm 5.03 \mathrm{D})$ was not statistically significant $(P=0.61)$.

Patients with $\geq 30$ years of age $(n=42)$ : The improvement in mean CDVA was not statistically significant (from $0.44 \pm 0.35 \log$ MAR, 20/55 Snellen, to $0.36 \pm 0.27$ logMAR, 20/45 Snellen, $P=0.074$ ) between baseline and 1 year, whereas maximum $K$ significantly decreased after CXL treatment (from $55.72 \pm 6.12 \mathrm{D}$ to $54.12 \pm 5.37 \mathrm{D}$, $P<0.001$ ).

Comparison of the postoperative changes in CDVA and maximum K between groups: After CXL treatment, the patients with $\geq 30$ years of age experienced more flattening in maximum $K$ compared with that of the patients with $<30$ years of age $(P=0.024)$, whereas there was no significant difference between two groups in terms of change in CDVA $(P=0.442)$.

Gender Males $(n=39)$ : The mean CDVA (from $0.35 \pm 0.25$ logMAR, 20/44 Snellen, to $0.27 \pm 0.22 \log$ MAR, 20/37 Snellen, $P=0.034$ ) and maximum $K$ (from $53.95 \pm 6.04 \mathrm{D}$ to $53.28 \pm 6.06 \mathrm{D}, P=0.005)$ significantly improved between baseline and 1 year after CXL treatment.

Table 1 Demographic data of the study group $(n=96)$

\begin{tabular}{lc}
\hline Characteristic & Value \\
\hline Gender & \\
Male, $n(\%)$ & $39(40.6)$ \\
Female, $n(\%)$ & $57(59.4)$ \\
Age, years (mean \pm SD) & $29.42 \pm 9.34$ \\
$18-29, n(\%)$ & $54(56.2)$ \\
$30-50, n(\%)$ & $42(43.8)$ \\
\hline
\end{tabular}

Females $(n=57)$ : There were significant improvements in mean CDVA (from 0.41 $\pm 0.32 \log M A R, 20 / 51$ Snellen, to $0.28 \pm 0.22 \log$ MAR, $20 / 38$ Snellen, $P=0.001)$ and maximum $K$ (from $54.94 \pm 5.18 \mathrm{D}$ to $53.69 \pm 4.53 \mathrm{D}$, $P=0.001)$ after CXL treatment.

Comparison of the postoperative changes in CDVA and maximum $K$ between groups: There were no significant differences regarding the changes in CDVA and maximum $K$ between males and females $(P=0.501$, $P=0.183$, respectively).

Preoperative CDVA CDVA $<0.3 \log M A R(n=39)$ : The mean CDVA did not show significant improvement (from $0.16 \pm 0.06 \log$ MAR, 20/28 Snellen, to $0.16 \pm 0.13$ logMAR, 20/28 Snellen, $P=0.905$ ), whereas the decrease in maximum $K$ was statistically significant (from $52.42 \pm 4.12 \mathrm{D}$ to $51.66 \pm 3.92 \mathrm{D}, P=0.002)$.

CDVA $\geq 0.3 \log M A R(n=57): \quad$ The mean CDVA (from $0.54 \pm 0.29 \log$ MAR, 20/69 Snellen, to $0.36 \pm 0.23$ logMAR, 20/45 Snellen, $P<0.001)$ and maximum $K$ significantly improved after CXL treatment (from 55.98 $\pm 5.94 \mathrm{D}$ to $54.80 \pm 5.57 \mathrm{D}, P=0.001$ ).

Comparison of the postoperative changes in CDVA and maximum $K$ between groups: The patients with a worse baseline visual acuity (CDVA $\geq 0.3 \log$ MAR) showed significant improvement in CDVA 1 year after CXL treatment compared with that of the patients with a better baseline visual acuity (CDVA $<0.3 \log M A R ; P<0.001)$. However, comparing two groups, the change in maximum $K$ was not statistically significant at 1 year $(P=0.316)$.

Preoperative maximum keratometry Maximum $\mathrm{K}<54 \mathrm{D}$ $(n=52)$ : The baseline CDVA (from $0.28 \pm 0.17 \log \mathrm{MAR}$, 20/38 Snellen, to $0.21 \pm 0.17 \log$ MAR, 20/32 Snellen, $P=0.009)$ and maximum $K$ significantly improved (from $50.54 \pm 2.10 \mathrm{D}$ to $49.74 \pm 2.31 \mathrm{D}, P=0.003$ ) after $\mathrm{CXL}$ treatment.

Maximum $K \geq 54 D(n=44)$ : After CXL treatment, mean CDVA (from $0.51 \pm 0.35 \log M A R, 20 / 64$ Snellen, to $0.35 \pm 0.25 \log$ MAR, 20/44 Snellen, $P=0.002)$ and maximum $K$ significantly improved (from $59.26 \pm 4.51 \mathrm{D}$ to $58.00 \pm 3.89 \mathrm{D}, P=0.003)$.

Table 2 Changes in visual acuity and topographic data between baseline and 1 year after CXL treatment in the entire cohort $(n=96)$

\begin{tabular}{lccr}
\hline & Baseline & 1 Year & P-value \\
\hline CDVA (logMAR, mean \pm SD) (Snellen equivalent) & $0.39 \pm 0.29(20 / 49)$ & $0.28 \pm 0.22(20 / 38)$ & $<0.001$ \\
Maximum keratometry (diopters, mean \pm SD) & $54.54 \pm 5.50$ & $53.52 \pm 5.18$ & $<\mathbf{0 . 0 0 1}$ \\
Thinnest pachymetry $(\mu \mathrm{m}$, mean \pm SD) & $460.11 \pm 47.15$ & $430.65 \pm 61.90$ & $<\mathbf{0 . 0 0 1}$ \\
\hline
\end{tabular}

Abbreviations: CDVA, corrected distance visual acuity; $\mathrm{CXL}$, corneal collagen crosslinking. Bold values indicate statistical significance $=P<0.05$, paired $t$-test. 
Comparison of the postoperative changes in CDVA and maximum $K$ between groups: There were no significant differences between two groups in terms of change in CDVA and maximum $K$ after CXL treatment $(P=0.099$, $P=0.332$, respectively).

Preoperative cone location. The study group comprised only eyes with central and paracentral cones.

Central cone $(n=72)$ : There were significant improvements in mean CDVA (from $0.43 \pm 0.32$ logMAR, 20/53 Snellen, to $0.30 \pm 0.23 \log$ MAR, 20/39 Snellen, $P<0.001$ ) and maximum $K$ (from $55.75 \pm 5.68 \mathrm{D}$ to $54.54 \pm 5.36 \mathrm{D}, P<0.001) 1$ year after $\mathrm{CXL}$ treatment.

Paracentral cone $(n=24)$ : There were no significant improvements in mean CDVA (from $0.24 \pm 0.09$ logMAR, 20/34 Snellen, to 0.21 $\pm 0.18 \log$ MAR, 20/32 Snellen, $P=0.239$ ) and maximum $K$ (from $50.90 \pm 2.92 \mathrm{D}$ to $50.47 \pm 3.05 \mathrm{D}, P=0.057$ ) at 1 -year follow-up.

Comparison of the postoperative changes in CDVA and maximum $K$ between the groups: The changes in mean CDVA and maximum $K$ after CXL treatment did not show significant differences between two groups ( $P=0.176, P=0.160$, respectively).

Preoperative thinnest pachymetry Thinnest pachymetry $<450 \mu m(n=45)$ : The baseline mean CDVA (from $0.44 \pm 0.35 \log$ MAR, 20/55 Snellen, to $0.32 \pm 0.23$ logMAR, 20/41 Snellen, $P=0.012$ ) and maximum $K$ (from 57.79 $\pm 5.52 \mathrm{D}$ to $56.10 \pm 5.17 \mathrm{D}, P<0.001$ ) significantly improved after CXL treatment.
Thinnest pachymetry $\geq 450 \mu m(n=51)$ : The mean CDVA (from $0.34 \pm 0.23 \log$ MAR, 20/43 Snellen, to $0.24 \pm 0.21$ logMAR, 20/34 Snellen, $P=0.001)$ significantly improved, whereas maximum $K$ (from $51.67 \pm 3.67 \mathrm{D}$ to $51.25 \pm 4.02 \mathrm{D}, P=0.124)$ did not show significant improvement after CXL treatment.

Comparison of the postoperative changes in CDVA and maximum $K$ between groups: The maximum $K$ significantly decreased in thinner corneas (pachymetry $<450 \mu \mathrm{m}$ ) compared with that of the relatively thicker corneas (pachymetry $\geq 450 \mu \mathrm{m} ; P=0.005$ ), whereas there was no significant difference between the groups with respect to improvement in CDVA $(P=0.627)$.

Postoperative changes in mean CDVA and maximum $K$ according to the preoperative factors are demonstrated in Figures 1 and 2.

\section{Discussion}

CXL using photosensitizer riboflavin and UVA-light has been introduced as a promising new treatment for progressive keratoconus to reduce the need for corneal transplantation. ${ }^{4}$ The treatment, as a photo-oxidative procedure, enhances biomechanical stability of the cornea because of synthesis of well-structured collagen and new lamellar interconnections in the cornea.5,6,15 Numerous clinical studies agreed that CXL treatment provides significant improvements in visual acuity and maximum keratometry as well as halting the progression of keratoconus. $7,8,16,17$ However, it is still unclear whether baseline demographic and topographic characteristics influence postoperative outcomes. Therefore, we sought effects of age, gender, preoperative CDVA, preoperative

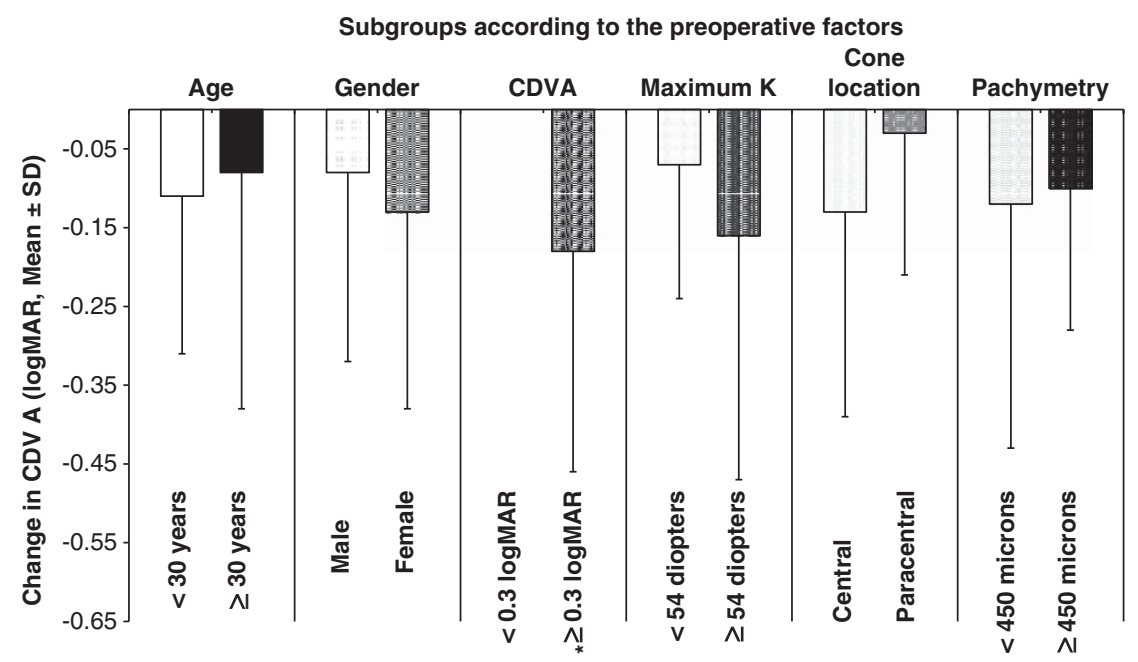

Figure 1 Change in mean corrected distance visual acuity (CDVA) between baseline and 1 year after CXL treatment in subgroups (*indicates statistically significant difference between two groups, $P<0.05$ ). 


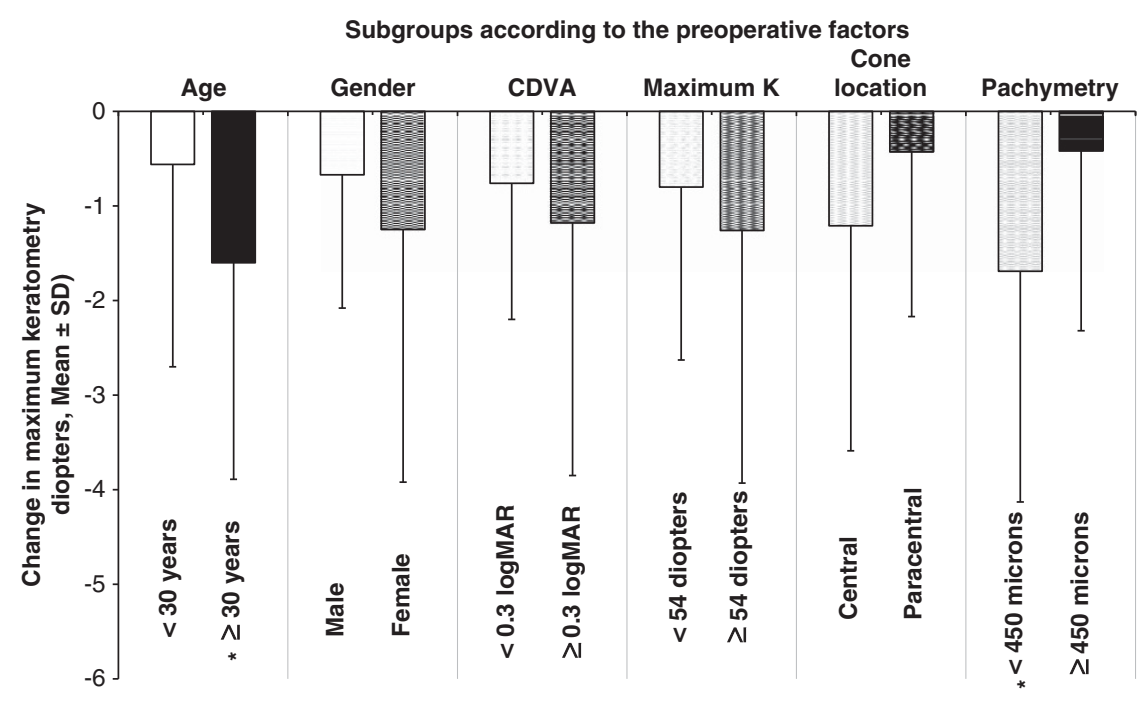

Figure 2 Mean decrease in maximum keratometry between baseline and 1 year in subgroups ( ${ }^{*}$ indicates statistically significant difference between two groups, $P<0.05)$.

maximum $K$, topographic cone location, and preoperative thinnest corneal pachymetry on improvements in CDVA and maximum $K$ at 1 year after CXL treatment. In the entire study group, the improvements in CDVA and maximum keratometry after CXL treatment were in agreement with the previous studies. ${ }^{9,10,18-20}$

In the current literature, a small number of studies investigated the effects of preoperative patient characteristics on outcomes of CXL treatment. Koller et $a l^{21}$ evaluated the effects of maximum $K$, preoperative CDVA, and age on complication and failure rates of CXL treatment for primary keratectasia. They suggested that age older than 35 years and a preoperative CDVA better than 20/25 (Snellen equivalent) were significant risk factors for complications (percentage of eyes losing two or more Snellen lines) and a preoperative maximum $K$ $>58 \mathrm{D}$ was identified a significant risk factor for failure (percentage of eyes with continued progression). On the contrary, another study by Koller et al ${ }^{22}$ demonstrated that a preoperative maximum $K$ of $>54.00 \mathrm{D}$ was a significant positive preoperative predictor of corneal flattening 1 year after CXL treatment. Similarly, a multifactorial analysis by Greenstein and colleagues revealed that patients with initial worse CDVA ( $\leq 20 / 40$ Snellen equivalent) and a higher keratometry ( $\geq 55 \mathrm{D})$ were most likely to benefit from CXL treatment. ${ }^{23}$

In our study, a preoperative CDVA $\leq 20 / 40$ Snellen equivalent was found significantly related with postoperative visual improvement. Furthermore, patients with an age $\geq 30$ years and a thinnest corneal pachymetry less than $450 \mu \mathrm{m}$ significantly experienced more flattening in maximum $K$. However, gender, preoperative maximum $\mathrm{K}$, and preoperative topographic cone location did not affect postoperative changes in mean CDVA and maximum $K$. Another study by Greenstein et al ${ }^{24}$ demonstrated more topographic flattening in eyes with centrally located cones compared with that of the peripherally located cones 1 year after CXL treatment, whereas differences among cone groups were not found statistically significant with respect to change in visual acuity.

It has been concluded that CXL is a safe and effective procedure for the treatment of progressive keratoconus, whereas it is still unclear which patients are more likely to benefit from the treatment. In clinical practice, it can be valuable to predict success of the treatment to give patients satisfactory information about postoperative course.

In conclusion, our study evaluated baseline demographic and topographic data to determine preoperative predictors of CXL treatment efficacy. Age, preoperative CDVA, and thinnest corneal pachymetry appear to influence the efficacy of CXL treatment.

\section{Summary}

What was known before

- Previous studies demonstrated the efficacy of corneal collagen crosslinking $(\mathrm{CXL})$ treatment in patients with keratoconus.

- Preoperative predictors of CXL efficacy were not completely elucidated.

What this study adds

- Patients with an older age ( $\geq 30$ years), a worse baseline corrected visual acuity ( $\leq 20 / 40$ Snellen equivalent), and a thinner baseline pachymetry $(<450 \mu \mathrm{m})$ most likely to benefit from CXL treatment.

- Preoperative corrected visual acuity was significantly related with visual improvement, whereas age and baseline thinnest corneal pachymetry showed a significant effect on flattening of maximum keratometry. 


\section{Conflict of interest}

The authors declare no conflict of interest.

\section{Acknowledgements}

We thank Birsen Gürkan and Süreyya Vural for supplying patient data. This study was performed in adherence to the tenets of the Declaration of Helsinki and Local Ethics Committee approved the study.

\section{References}

1 Rabinowitz YS. Keratoconus. Surv Ophthalmol 1998; 42: 297-319.

2 Krachmer JH, Feder RS, Belin MW. Keratoconus and related noninflammatory corneal thinning disorders. Surv Ophthalmol 1984; 28: 293-322.

3 Wollensak G, Spoerl E, Seiler T. Riboflavin/ultravioletA-induced collagen crosslinking for the treatment of keratoconus. Am J Ophthalmol 2003; 135: 620-627.

4 Wollensak G. Crosslinking treatment of progressive keratoconus: new hope. Curr Opin Ophthalmol 2006; 17: 356-360.

5 Spoerl E, Wollensak G, Seiler T. Increased resistance of crosslinked cornea against enzymatic digestion. Curr Eye Res 2004; 29: 35-40.

6 Wollensak G, Spoerl E, Seiler T. Stress-strain measurements of human and porcine corneas after riboflavin-ultraviolet-Ainduced cross-linking. J Cataract Refract Surg 2003; 29: 1780-1785.

7 Caporossi A, Baiocchi S, Mazzotta C, Traversi C, Caporossi T. Parasurgical therapy for keratoconus by riboflavin-ultraviolet type A rays induced cross-linking of corneal collagen; preliminary refractive results in an Italian study. J Cataract Refract Surg 2006; 32: 837-845.

8 Raiskup-Wolf F, Hoyer A, Spoerl E, Pillunat LE. Collagen crosslinking with riboflavin and ultraviolet-A light in keratoconus: long-term results. J Cataract Refract Surg 2008; 34: 796-801.

9 Caporossi A, Mazzotta C, Baiocchi S, Caporossi T. Long-term results of riboflavin ultraviolet A corneal collagen cross-linking for keratoconus in Italy: the Siena Eye Cross Study. Am J Ophthalmol 2010; 149: 585-593.

10 Hersh PS, Greenstein SA, Fry KL. Corneal collagen crosslinking for keratoconus and corneal ectasia: one-year results. J Cataract Refract Surg 2011; 37: 149-160.

11 Holladay JT, Prager TC. Mean visual acuity. Am J Ophthalmol 1991 111: 372-374

12 Yaylali V, Kaufman SC, Thompson HW. Corneal thickness measurements with the Orbscan Topography System and ultrasonic pachymetry. J Cataract Refract Surg 1997; 23: 1345-1350.

13 Amano S, Honda N, Amano Y, Yamagami S, Miyai T, Samejima T et al. Comparison of central corneal thickness measurements by rotating Scheimpflug camera, ultrasonic pachymetry, and scanning-slit corneal topography. Ophthalmology 2006; 113: 937-941.

14 de Sanctis U, Missolungi A, Mutani B, Richiardi L, Grignolo FM. Reproducibility and repeatability of central corneal thickness measurement in keratoconus using the rotating Scheimpflug camera and ultrasound pachymetry. Am J Ophthalmol 2007; 144: 712-718.

15 Mazzotta C, Balestrazzi A, Traversi C, Baiocchi S, Caporossi T, Tommasi $\mathrm{C}$ et al. Treatment of progressive keratoconus by riboflavin-UVA-induced cross-linking of corneal collagen; ultrastructural analysis by Heidelberg Retinal Tomograph II in vivo confocal microscopy in humans. Cornea 2007; 26: 390-397.

16 Grewal DS, Brar GS, Jain R, Sood V, Singla M, Grewal SPS. Corneal collagen crosslinking using riboflavin and ultraviolet-A light for keratoconus; one-year analysis using Scheimpflug imaging. J Cataract Refract Surg 2009; 35: 425-432.

17 Legare ME, Iovieno A, Yeung SN, Kim P, Lichtinger A, Hollands $\mathrm{S}$ et al. Corneal collagen cross-linking using riboflavin and ultraviolet $\mathrm{A}$ for the treatment of mild to moderate keratoconus: 2-year follow-up. Can J Ophthalmol 2013; 48: 63-68.

18 O'Brart DP, Kwong TQ, Patel P, McDonald RJ, O'Brart NA. Long-term follow-up of riboflavin/ultraviolet A (370 nm) corneal collagen cross-linking to halt the progression of keratoconus. Br J Ophthalmol 2013; 97: 433-437.

19 Vinciguerra P, Albè E, Trazza S, Rosetta P, Vinciguerra R, Seiler $\mathrm{T}$ et al. Refractive, topographic, tomographic, and aberrometric analysis of keratoconic eyes undergoing corneal cross-linking. Ophthalmology 2009; 116: 369-378.

20 Vinciguerra P, Camesasca FI, Albè E, Trazza S. Corneal collagen cross-linking for ectasia after excimer laser refractive surgery: 1-year results. J Refract Surg 2010; 26: 486-497.

21 Koller T, Mrochen M, Seiler T. Complication and failure rates after corneal cross-linking. J Cataract Refract Surg 2009; 35: 1358-1362.

22 Koller T, Pajic B, Vinciguerra P, Seiler T. Flattening of the cornea after collagen cross-linking for keratoconus. J Cataract Refract Surg 2011; 37: 1488-1492.

23 Greenstein S, Hersh PS. A multifactorial treatment analysis and algorithm for corneal collagen cross-linking http:/ / www.arvo.org. Association for Research in Vision and Ophthalmology Web site (accessed 2013).

24 Greenstein SA, Fry KL, Hersh PS. Effect of topographic cone location on outcomes of corneal collagen cross-linking for keratoconus and corneal ectasia. J Refract Surg 2012; 28: 397-405. 